\title{
Frequency and Spectrum of KRAS Mutations in Moroccan Patients with Lung Adenocarcinoma
}

\author{
Ibrahim Elghissassi, ${ }^{1}$ Hanane Inrhaoun, ${ }^{1}$ Anwar Boukir, ${ }^{1}$ Fouad Kettani, ${ }^{2}$ Lamia Gamra, \\ Amina Mestari, ${ }^{4}$ Lamia Jabri, ${ }^{5}$ Youssef Bensouda, ${ }^{1}$ Hind Mrabti, ${ }^{1}$ and Hassan Errihani ${ }^{1}$ \\ ${ }^{1}$ Medical Oncology Department, National Institute of Oncology, Allal Elfassi Street, 62000 Rabat, Morocco \\ ${ }^{2}$ Pathology Department, Nations-Unies Pathology Center, Rabat, Morocco \\ ${ }^{3}$ Pathology Department, Hassan Pathology Center, Rabat, Morocco \\ ${ }^{4}$ Pathology Department, Agdal Pathology Center, Rabat, Morocco \\ ${ }^{5}$ Pathology Department, Casapath Pathology Center, Casablanca, Morocco
}

Correspondence should be addressed to Ibrahim Elghissassi; i_elghissassi@hotmail.com

Received 23 December 2013; Accepted 4 February 2014; Published 5 March 2014

Academic Editors: G. Chen, S. Holdenrieder, H. Mo, and H. Zhang

Copyright (C) 2014 Ibrahim Elghissassi et al. This is an open access article distributed under the Creative Commons Attribution License, which permits unrestricted use, distribution, and reproduction in any medium, provided the original work is properly cited.

Background. In lung adenocarcinoma, the frequency of KRAS mutations is ethnicity dependent with a higher proportion in African Americans and white Caucasians than in Asians. The prevalence of these mutations among North Africans patients is unknown. The objective of this study was to report the frequency and spectrum of KRAS mutations in a group of Moroccan lung adenocarcinoma patients. Methods. Tumor specimens from 117 Moroccan patients with lung adenocarcinoma were selected to determine frequency and spectrum of KRAS mutations. KRAS mutations in codons 12 and 13 of exon 2 were analyzed using conventional DNA sequencing. Results. The overall frequency of the KRAS mutations was 9\% (11/117). In the population with KRAS mutations, there was a trend towards more male $(P=0.06)$ and more smokers $(P=0.08)$ compared to patients with wild type KRAS. KRAS mutations were located at codon 12 in 10 out of 11 patients (91\%). The G12C mutation was the most frequent KRAS mutation (73\%). Conclusion. This is the first study to date examining the frequency and spectrum of KRAS mutations in lung adenocarcinomas in North African and Arab populations. KRAS mutation frequency in Moroccan patients was comparable with the frequency observed in East-Asian population. KRAS mutations are more likely observed in males and smokers and to be transversions. Further studies, in larger numbers of patients, are needed to confirm these findings.

\section{Introduction}

Lung cancer is one of the most common cancers in Morocco and in the world and is the leading cause of cancer mortality in both males and females $[1,2]$. During the last few years, improvement in the knowledge of lung cancer molecular biology led to identification of several biological events crucial for tumor cell survival.

In nonsmall-cell lung cancer (NSCLC), one of the most commonly genetic aberrations is conversion of the protooncogene KRAS to its activated oncogenic form. KRAS encodes low molecular weight GTPase binding proteins that regulate cell growth, differentiation, and apoptosis. Mutations of KRAS are associated with impaired GTPase activity, causing increased mitogenic RAS signaling [3].

Mutations in KRAS gene occur more frequently (20$30 \%$ ) in adenocarcinoma and less frequently (about 7\%) in squamous-cell carcinoma and are usually associated with a history of smoking [4]. The incidence of KRAS mutation is ethnicity dependent with a higher proportion in African Americans and white Caucasians $(20-30 \%)$ than in Asians (5-20\%) [5-7]. However, such information is still lacking in some other races, such as North African patients.

Typically, KRAS mutations are a point mutation in the gene replacing an amino acid at positions 12,13 , or 61 [8]. Somatic KRAS mutations predominantly involve codons 12 
and 13 . Together, these two mutations account for nearly $95 \%$ of all KRAS mutations in lung cancer. The G12C mutation in codon 12 is by far the most frequent mutation in NSCLC [8$10]$.

The main goal of this study was to report the frequency and spectrum of KRAS mutations in unselected group of Moroccan lung adenocarcinoma patients. In addition, we have examined the relationship between the mutations and some parameters, including gender and smoking status.

\section{Methods}

2.1. Patients and Tumor Samples. Data of all lung adenocarcinoma samples were collected from four pathology laboratories in Morocco between November 2010 and August 2012. The participating laboratories were Nations-Unies Pathology Center, Rabat; Hassan Pathology Center, Rabat; Agdal Pathology Center, Rabat; Casapath Pathology Center, Casablanca.

All of these laboratories outsource KRAS mutation testing (Molecular Biology Laboratory in Paris, France) and perform more than $90 \%$ of all tests requested in Morocco.

Only Moroccan metastatic lung adenocarcinoma patients were included. The histological diagnosis of adenocarcinoma was verified using the pathology report provided by participating laboratories.

Never smokers were defined as individuals who reported smoking fewer than 100 cigarettes in their lifetime.

2.2. KRAS Mutation Analysis. Genomic DNA was extracted from tumors tissues according to standard procedures. KRAS mutations in codons 12 and 13 of exon 2 were analyzed using conventional DNA sequencing.

2.3. Ethics Statement. The ethics committee approval was not necessary for this study, since all mutation tests were part of the routine diagnostic procedure and all patient and tumor characteristics were collected anonymously.

2.4. Statistical Analysis. The potential associations of KRAS mutation with age, gender, and smoking status were analyzed using Fisher's exact test. Means of age were compared using the $T$ test. A $P$ value less than 0.05 was considered statistically significant. All analyses were performed using SPSS Version 20.

\section{Results}

One hundred and seventeen lung adenocarcinomas from Moroccan patients were collected. The study population consisted of 77 men (66\%) and 40 women (34\%), with a median age of 59 years (range: 37 to 87 years). The demographic characteristics of the patients are provided in Table 1.

A total of 11 KRAS mutations were found in this series of 117 patients (9\%). KRAS mutations were identified in 13\% of smokers (9/68) and $4 \%$ of never smokers (2/49). There were $13 \%$ of male $(10 / 77)$ and $2 \%$ of female $(1 / 40)$ with KRAS mutations.
TABLE 1: Demographic characteristics of the study population.

\begin{tabular}{lcc}
\hline & \multicolumn{2}{c}{ All patients $(n=117)$} \\
& No & \\
\hline Age (years) & 59 & \\
$\quad$ Median & $37-87$ & \\
Range & & $(66 \%)$ \\
Sex & 77 & $(34 \%)$ \\
$\quad$ Male & 40 & $(42 \%)$ \\
Female & & $(58 \%)$ \\
Smoking history & 49 & \\
$\quad$ Never & 68 & $(100 \%)$ \\
$\quad$ Ever & & \\
Histology & 117 & $(100 \%)$ \\
$\quad$ Adenocarcinoma & & \\
Stage at diagnosis & 117 & \\
$\quad$ IV & &
\end{tabular}

In the population with KRAS mutations, there was a trend towards more male than female compared to patients with wild type KRAS ( $91 \%$ versus $63 \%, P=0.06$ ) but this did not reach statistical significance. The frequency of smokers in patients with tumors having KRAS mutations was higher than that observed in patients without mutations $(82 \%$ versus $56 \%, P=0.08$ ) but also this did not reach statistical significance. The mean age did not differ between the two groups (Table 2).

All KRAS mutations were located at codon 12, with the exception of a single patient in which KRAS was mutated at codon 13. The G12C mutation was the most frequent KRAS mutation (73\%) in this series (Table 3).

\section{Discussion}

In the present series, KRAS mutations were identified in 11 of 117 Moroccan patients (9\%). To the best of our knowledge, this study represents the first study to date reporting KRAS mutation rate in North African and Arabs populations.

Some previous studies have demonstrated a genetic divergence of KRAS mutation rates according to ethnicity [1118]. Indeed, KRAS mutations rate in Caucasian (26-37\%) and African-American (17-37\%) patients is much higher than that in East-Asian population (6-11\%). KRAS mutation frequency in Moroccan patients, as reported in this study, was comparable with the frequency observed in East-Asian population (Table 4).

In the population with KRAS mutations, we found a trend towards more male than female compared to patients with wild type KRAS (91\% versus $63 \%, P=0.06$ ). This did not reach statistical significance, probably due to the low number of patients with KRAS mutations in the present study. However, these results are in keeping with some published reports showing a trend towards more mutations in males $[19,20]$. 
TABLE 2: Patient characteristics by KRAS mutation status.

\begin{tabular}{|c|c|c|c|c|c|}
\hline & \multicolumn{2}{|c|}{ KRAS mutations $(n=11)$} & \multicolumn{2}{|c|}{ Wild type $(n=106)$} & \multirow{2}{*}{$P$} \\
\hline & No & $\%$ & No & $\%$ & \\
\hline Mean age at diagnosis & 60 & & 60 & & $P=0.98^{*}$ \\
\hline \multicolumn{6}{|l|}{ Sex } \\
\hline Male & 10 & $(91 \%)$ & 67 & $(63 \%)$ & \multirow{2}{*}{$P=0.06^{* *}$} \\
\hline Female & 1 & $(9 \%)$ & 39 & $(37 \%)$ & \\
\hline \multicolumn{6}{|l|}{ Smoking history } \\
\hline Ever & 9 & $(82 \%)$ & 59 & $(56 \%)$ & \multirow[t]{2}{*}{$P=0.08^{* *}$} \\
\hline Never & 2 & $(18 \%)$ & 47 & $(44 \%)$ & \\
\hline
\end{tabular}

${ }^{*} P$ value calculated by $t$-test.

${ }^{* *} P$ value calculated by Fisher's exact test.

TABLE 3: Frequency of KRAS mutation type.

\begin{tabular}{ccccc}
\hline \multicolumn{2}{c}{ KRAS } & \multirow{2}{*}{ Smokers } & Never smokers & Total (\%) \\
Mutation & Nucleotide & & & \\
\hline Codon 12 & & & & \\
G12C & GGT $\rightarrow$ TGT & 8 & 0 & $8(73 \%)$ \\
G12S & GGT $\rightarrow$ AGT & 1 & 0 & $1(9 \%)$ \\
G12D & GGT $\rightarrow$ GAT & 0 & 1 & $1(9 \%)$ \\
Codon 13 & & & & \\
G13D & GGC $\rightarrow$ GAC & 0 & 1 & $1(9 \%)$ \\
\hline
\end{tabular}

TABLE 4: The frequency of KRAS mutations by race.

\begin{tabular}{lcc}
\hline Study & Race & KRAS Mutation (\%) \\
\hline Smits et al. [11] & Caucasian & $244 / 661(37 \%)$ \\
Boch et al. [12] & Caucasian & $67 / 254(26 \%)$ \\
Reinersman et al. [13] & Caucasian & $125 / 476(26 \%)$ \\
Hunt et al. [14] & African-American & $22 / 60(37 \%)$ \\
Leidner et al. [15] & African-American & $12 / 53(23 \%)$ \\
Reinersman et al. [13] & African-American & $21 / 121(17 \%)$ \\
Sasaki et al. [16] & East Asian & $21 / 190(11 \%)$ \\
Kim et al. [17] & East Asian & $5 / 71(7 \%)$ \\
Akamatsu et al. [18] & East Asian & $2 / 31(6 \%)$ \\
This study & North African & $11 / 117(9 \%)$ \\
\hline
\end{tabular}

In this study, the higher frequency of KRAS mutations among smokers (82\%) supports data from some previous studies. For example, Ahrendt et al. reported a strong positive association between presence of KRAS mutations and smoking [21]. A meta-analysis of KRAS mutations in NSCLC reported a frequency of $26 \%$ in tumors of current/former smokers and $6 \%$ in tumors of never smokers [22]. On the other hand, KRAS mutations have been detected in a significant proportion of never smoker lung adenocarcinoma patients, with an incidence up to $15 \%$ [7], and thus remain not rare in this subpopulation.

In lung adenocarcinomas, the majority of KRAS mutations have been identified in codons 12 and 13. In our subjects, the distribution of mutations of KRAS at codons $12(10 / 11$ $(91 \%))$ and $13(1 / 11(9 \%))$ appear to be comparable with the frequency of the other studies $[23,24]$. Furthermore, the
G12C mutation was the most frequent KRAS mutation (73\%) confirming previous results [8-10].

Transversions (substituting a pyrimidine base for a purine base or vice versa) are more common than transitions (substituting purine for purine or pyrimidine for pyrimidine). KRAS mutations observed in smokers are more likely to be transversions unlike the transitions more common in patients with no history of cigarette smoking [7]. Indeed, the etiology of $\mathrm{G} \rightarrow \mathrm{T}$ transversion in lung cancer has been attributed to the exposure to polycyclic aromatic hydrocarbons (PAH) present in tobacco smoke [25]. In the present study, $89 \%$ of smokers (8/9) had a transversion mutation supporting these data.

Till date, the clinical applicability of the KRAS mutation is limited. Firstly, there is no specific treatment currently available for NSCLC with KRAS mutations. Secondly, the prognostic and predictive role of KRAS mutations in NSCLC remains controversial. While some studies suggested that mutant KRAS may have a potential negative prognostic effect and could be predictive of lack of response to chemotherapy and epidermal growth factor receptor (EGFR) inhibitors, other studies failed to confirm this result $[6,26]$. However, previous studies have shown that EGFR and KRAS mutations are mutually exclusive $[5,19]$. KRAS may therefore have a role as part of an efficient testing algorithm.

In Summary, the present study revealed that KRAS mutation frequency in Moroccan patients was comparable with the frequency observed in East-Asian population. In keeping with the literature, KRAS mutations are more likely observed in males and smokers and to be transversions. Further studies, in larger numbers of patients, are needed to confirm these findings.

\section{Conflict of Interests}

All authors declare that they have no competing interests.

\section{Authors' Contribution}

Ibrahim Elghissassi drafted the paper; Hanane Inrhaoun helped to draft the paper; Anwar Boukir, Fouad Kettani, Lamia Gamra, Amina Mestari, and Lamia Jabri participated in acquisition of data; Youssef Bensouda, Hind Mrabti, and 
Hassan Errihani have been involved in revising the paper. All authors read and approved the final paper.

\section{Acknowledgment}

This work was supported by the Research and Investigation in Medical Oncology Moroccan Group.

\section{References}

[1] R. Siegel, D. Naishadham, and A. Jemal, "Cancer statistics, 2013," CA Cancer Journal for Clinicians, vol. 63, pp. 11-30, 2012.

[2] The Grand Casablanca cancer registry, 2007.

[3] S. L. Campbell, R. Khosravi-Far, K. L. Rossman, G. J. Clark, and C. J. Der, "Increasing complexity of Ras signaling," Oncogene, vol. 17, no. 11, pp. 1395-1413, 1998.

[4] S. Forbes, J. Clements, E. Dawson et al., “Cosmic 2005,” British Journal of Cancer, vol. 94, no. 2, pp. 318-322, 2006.

[5] M. Suzuki, H. Shigematsu, T. Iizasa et al., "Exclusive mutation in epidermal growth factor receptor gene, HER-2, and KRAS, and synchronous methylation of nonsmall cell lung cancer," Cancer, vol. 106, no. 10, pp. 2200-2207, 2006.

[6] R. Califano, L. Landi, and F. Cappuzzo, "Prognostic and predictive value of K-RAS mutations in non-small cell lung cancer," Drugs, vol. 72, no. 1 supplement, pp. 28-36, 2012.

[7] G. J. Riely, M. G. Kris, D. Rosenbaum et al., "Frequency and distinctive spectrum of KRAS mutations in never smokers with lung adenocarcinoma," Clinical Cancer Research, vol. 14, no. 18, pp. 5731-5734, 2008.

[8] J. L. Bos, "Ras oncogenes in human cancer: a review," Cancer Research, vol. 49, no. 17, pp. 4682-4689, 1989.

[9] L. Ding, G. Getz, D. A. Wheeler et al., "Somatic mutations affect key pathways in lung adenocarcinoma," Nature, vol. 455, no. 7216, pp. 1069-1075, 2008.

[10] S. Schubbert, K. Shannon, and G. Bollag, "Hyperactive Ras in developmental disorders and cancer," Nature Reviews Cancer, vol. 7, no. 4, pp. 295-308, 2007.

[11] A. J. Smits, J. A. Kummer, J. W. Hinrichs et al., "EGFR and KRAS mutations in lung carcinomas in the Dutch population: increased EGFR mutation frequency in malignant pleural effusion of lung adenocarcinoma," Cellular Oncology, vol. 35, no. 3, pp. 189-196, 2012.

[12] C. Boch, J. Kollmeier, A. Roth et al., "The frequency of EGFR and KRAS mutations in non-small cell lung cancer (NSCLC): routine screening data for central Europe from a cohort study," BMJ Open, vol. 3, no. 4, Article ID e002560, 2013.

[13] J. M. Reinersman, M. L. Johnson, G. J. Riely et al., "Frequency of EGFR and KRAS mutations in lung adenocarcinomas in African Americans," Journal of Thoracic Oncology, vol. 6, no. 1, pp. 28-31, 2011.

[14] J. D. Hunt, A. Strimas, J. E. Martin et al., "Differences in KRAS mutation spectrum in lung cancer cases between African Americans and Caucasians after occupational or environmental exposure to known carcinogens," Cancer Epidemiology Biomarkers and Prevention, vol. 11, no. 11, pp. 1405-1412, 2002.

[15] R. S. Leidner, P. Fu, B. Clifford et al., "Genetic abnormalities of the EGFR pathway in African American patients with nonsmall-cell lung cancer," Journal of Clinical Oncology, vol. 27, no. 33, pp. 5620-5626, 2009.
[16] H. Sasaki, K. Okuda, O. Kawano et al., "Nras and Kras mutation in Japanese lung cancer patients: genotyping analysis using LightCycler," Oncology Reports, vol. 18, no. 3, pp. 623-628, 2007.

[17] Y. T. Kim, T.-Y. Kim, D. S. Lee et al., "Molecular changes of epidermal growth factor receptor (EGFR) and KRAS and their impact on the clinical outcomes in surgically resected adenocarcinoma of the lung," Lung Cancer, vol. 59, no. 1, pp. 111-118, 2008.

[18] H. Akamatsu, K. Kaira, H. Murakami et al., "The impact of clinical outcomes according to EGFR mutation status in patients with locally advanced lung adenocarcinoma who recieved concurrent chemoradiotherapy," American Journal of Clinical Oncology, 2012.

[19] S. Y. Lee, M. J. Kim, G. Jin et al., "Somatic mutations in epidermal growth factor receptor signaling pathway genes in nonsmall cell lung cancers," Journal of Thoracic Oncology, vol. 5, no. 11, pp. 1734-1740, 2010.

[20] K. Takamochi, S. Oh, and K. Suzuki, "Differences in EGFR and KRAS mutation spectra in lung adenocarcinoma of never and heavy smokers," Oncology Letters, vol. 6, no. 5, pp. 1207-1212, 2013.

[21] S. A. Ahrendt, P. A. Decker, E. A. Alawi et al., "Cigarette smoking is strongly associated with mutation of the K-ras gene in patients with primary adenocarcinoma of the lung," Cancer, vol. 92, no. 6, pp. 1525-1530, 2001.

[22] C. Mao, L.-X. Qiu, R.-Y. Liao et al., "KRAS mutations and resistance to EGFR-TKIs treatment in patients with non-small cell lung cancer: a meta-analysis of 22 studies," Lung Cancer, vol. 69, no. 3, pp. 272-278, 2010.

[23] T. S. Mok, Y.-L. Wu, S. Thongprasert et al., "Gefitinib or carboplatin-paclitaxel in pulmonary adenocarcinoma," The New England Journal of Medicine, vol. 361, no. 10, pp. 947-957, 2009.

[24] J. M. Siegfried, A. T. Gillespie, R. Mera et al., "Prognostic value of specific KRAS mutations in lung adenocarcinomas," Cancer Epidemiology Biomarkers and Prevention, vol. 6, no. 10, pp. 841847, 1997.

[25] S. S. Hecht, "Tobacco smoke carcinogens and lung cancer," Journal of the National Cancer Institute, vol. 91, no. 14, pp. 1194-1210, 1999.

[26] P. Martin, N. B. Leighl, M. S. Tsao, and F. A. Shepherd, "KRAS mutations as prognostic and predictive markers in non-small cell lung cancer," Journal of Thoracic Oncology, vol. 8, no. 5, pp. 530-542, 2013. 


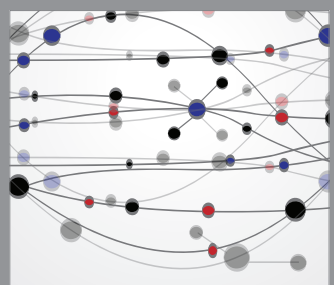

The Scientific World Journal
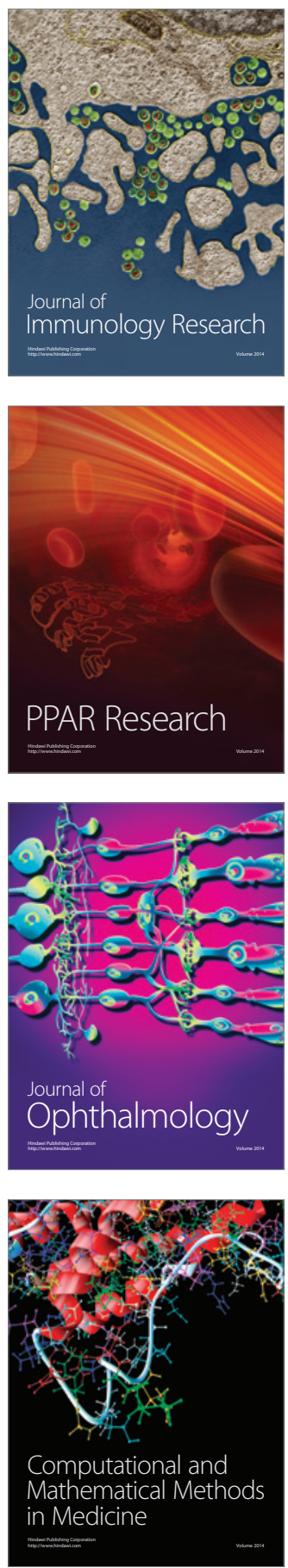

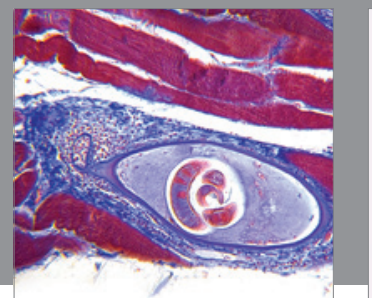

Gastroenterology

Research and Practice
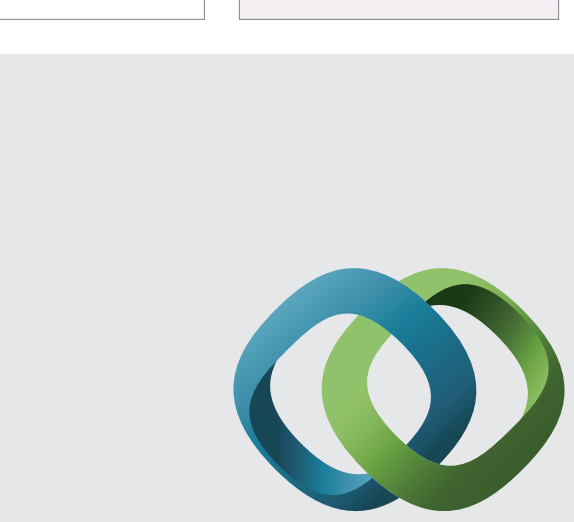

\section{Hindawi}

Submit your manuscripts at

http://www.hindawi.com
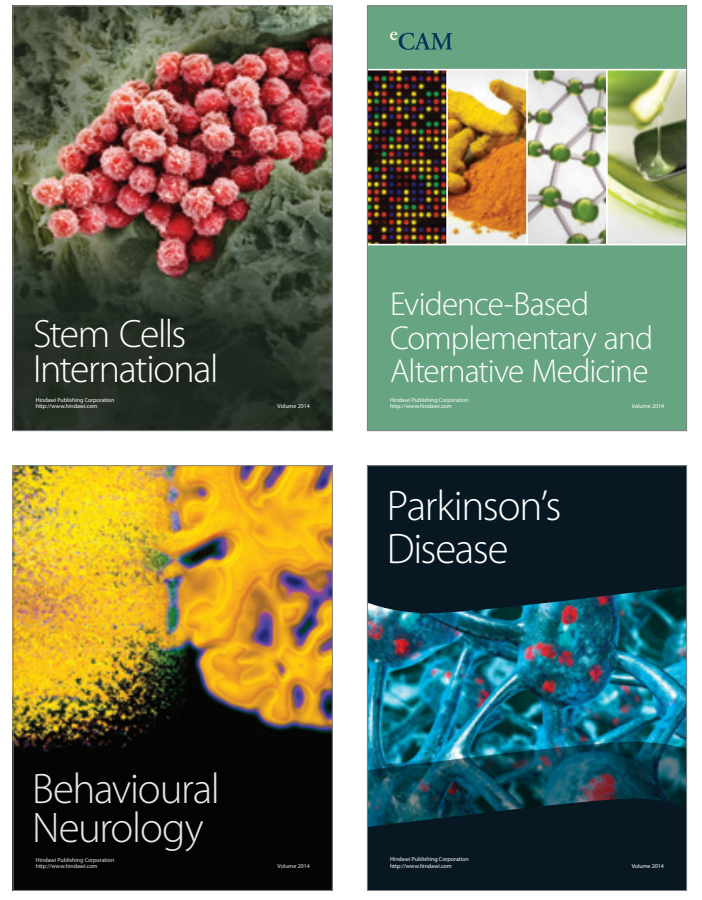
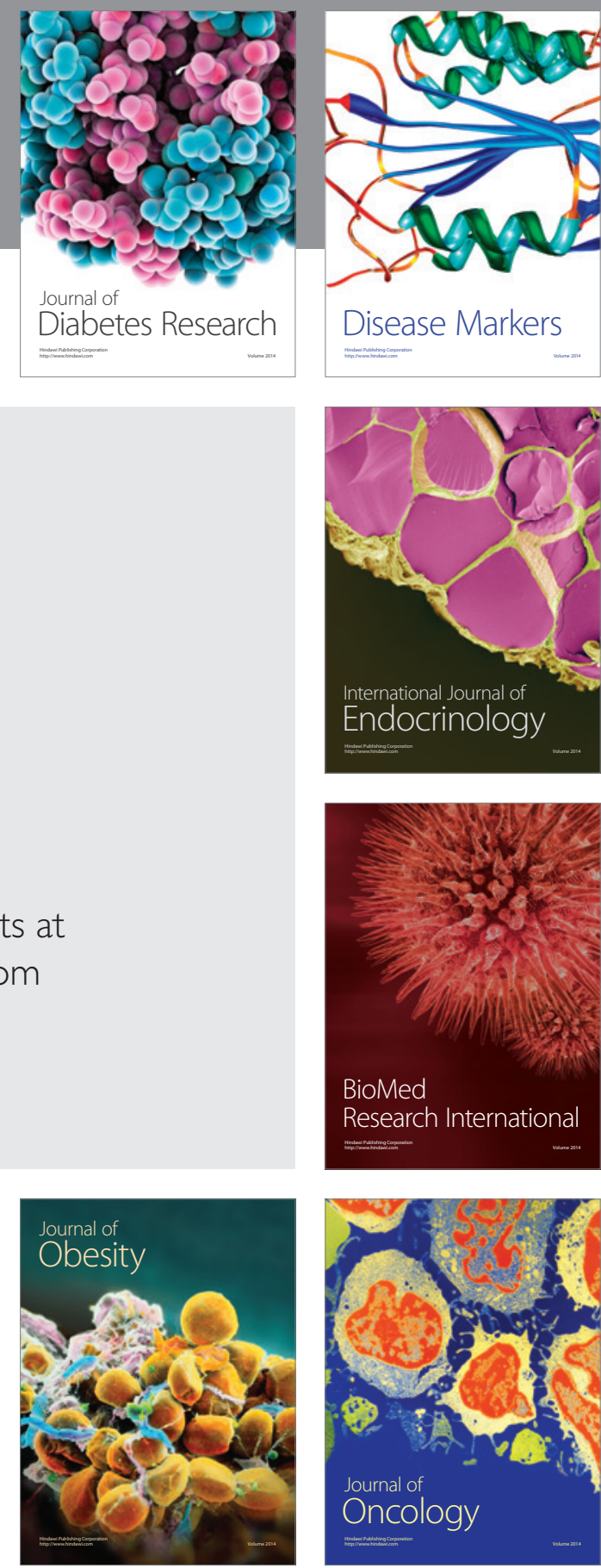

Disease Markers
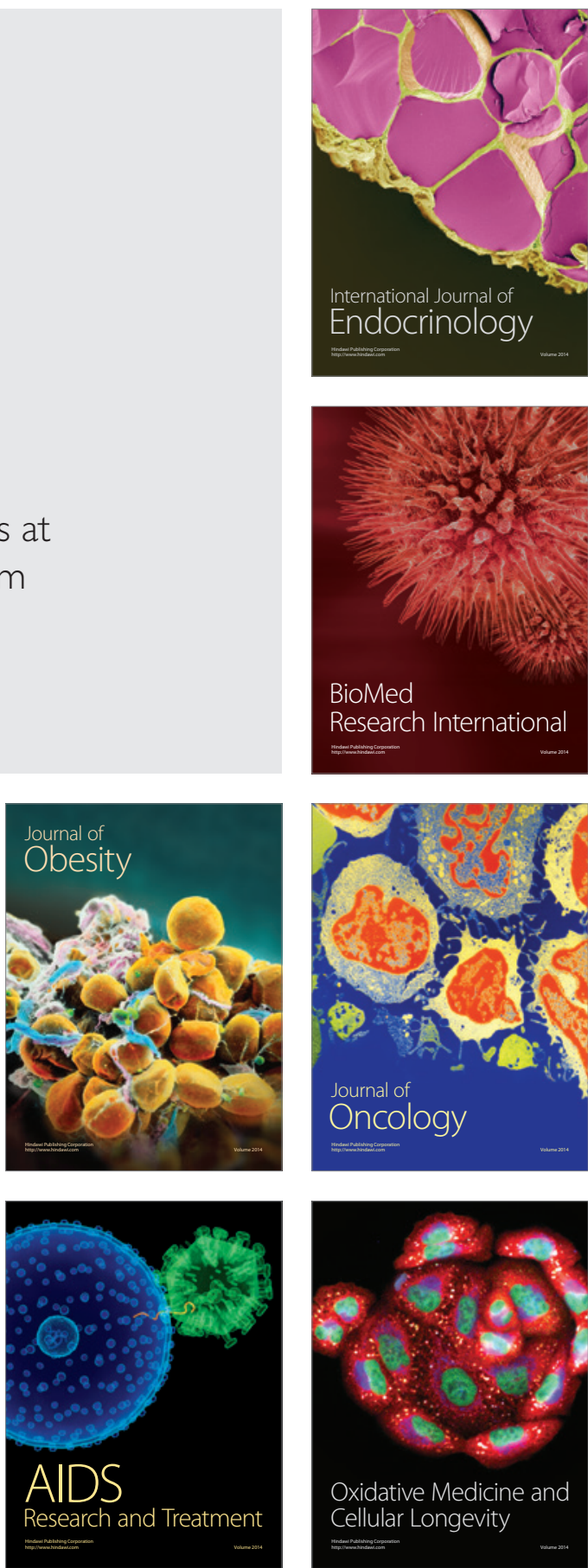